\title{
Optimal Parametrization of Deviations from Tribimaximal Form of the Neutrino Mass Matrix
}

\author{
Christopher D. Carone* \\ Department of Physics, College of William \& Mary, \\ Williamsburg, VA 23187-8795, USA \\ Richard F. Lebed $\dagger$ \\ Department of Physics, Arizona State University, Tempe, AZ 85287-1504, USA
}

(Dated: October, 2009)

\begin{abstract}
We obtain a general parametrization for the neutrino Majorana mass matrix in which all possible deviations from tribimaximal mixing are given by three complex parameters, and the deviation from each tribimaximal mixing eigenvector is identified with precisely two of them. This parametrization provides a useful tool for classifying the corrections to exact tribimaximal mixing in flavor models.
\end{abstract}

PACS numbers: 14.60.Pq, 12.15.Ff, 11.30.Hv

*Electronic address: cdcaro@wm.edu

${ }^{\dagger}$ Electronic address: Richard.Lebed@asu.edu 
Introduction. Neutrino mass and mixing data to date [1] strongly favor a "tribimaximal" (TB) mixing [2] relationship between the neutrino mass and flavor eigenstates. The unitary TB neutrino mixing matrix is given by

$$
U_{\mathrm{TB}}=\left(\begin{array}{ccc}
\sqrt{\frac{2}{3}} & \frac{1}{\sqrt{3}} & 0 \\
-\frac{1}{\sqrt{6}} & \frac{1}{\sqrt{3}} & -\frac{1}{\sqrt{2}} \\
-\frac{1}{\sqrt{6}} & \frac{1}{\sqrt{3}} & +\frac{1}{\sqrt{2}}
\end{array}\right),
$$

assuming a conventional choice of phases. $U_{\mathrm{TB}}$ is the special case of the general (Pontecorvo)Maki-Nakagawa-Sakata (MNS) [3] form

$$
U_{\mathrm{MNS}}=\left(\begin{array}{ccc}
c_{13} c_{12} e^{i \beta_{1}} & c_{13} s_{12} e^{i \beta_{2}} & s_{13} e^{-i \delta} \\
\left(-s_{12} c_{23}+s_{13} c_{12} s_{23} e^{i \delta}\right) e^{i \beta_{1}} & \left(c_{12} c_{23}+s_{13} s_{12} s_{23} e^{i \delta}\right) e^{i \beta_{2}} & -c_{13} s_{23} \\
\left(-s_{12} s_{23}-s_{13} c_{12} c_{23} e^{i \delta}\right) e^{i \beta_{1}} & \left(c_{12} s_{23}-s_{13} s_{12} c_{23} e^{i \delta}\right) e^{i \beta_{2}} & c_{13} c_{23}
\end{array}\right)
$$

where $s_{i j} \equiv \sin \theta_{i j}, c_{i j} \equiv \cos \theta_{i j}$, for which $s_{13}=0$ (and hence $\delta$ is irrelevant), $s_{12}=1 / \sqrt{3}$ and $c_{12}=\sqrt{2 / 3}, s_{23}=c_{23}=1 / \sqrt{2}$, and the Majorana phases $\beta_{1,2}$, which do not contribute to neutrino oscillation processes, are set to zero. While deviations from TB mixing can be described in terms of the angles of the MNS matrix, it is more convenient to work directly with the eigenvectors of the neutrino mass matrix (i.e., the columns of $U_{\mathrm{TB}}$ ), which do not depend on the conventions for parametrizing unitary matrices.

The purpose of this note is simply to show that deviations from the tribimaximal form of the neutrino Majorana mass matrix are given uniquely by a set of three independent complex parameters with the property that setting any two of them to zero preserves one of the vectors forming the columns of $U_{\mathrm{TB}}$. Moreover, this property remains true even when the nonzero parameter is arbitrarily large. These special parameters are easily identified by the specific form of the neutrino mass matrix in any given model. In the physical case, for which all the deviations from TB mixing are small, the conventional angles and phases of the MNS matrix all have simple forms at linear order in these parameters. We compare our approach to other relevant parametrizations at the end of the discussion.

Diagonalization. The MNS matrix brings the neutrino Majorana mass matrix $M_{L L}$ to normal form, $\operatorname{diag}\left(m_{1} m_{2} m_{3}\right)=U_{\mathrm{MNS}}^{T} M_{L L} U_{\mathrm{MNS}}$, with $m_{i} \geq 0$. In general, $M_{L L} \equiv M$ is a complex symmetric matrix, meaning that its "diagonalization" by means of eigenvectors is not completed by the usual means (as evidenced by the presence of $U_{\text {MNS }}^{T}$ rather than $U_{\text {MNS }}^{\dagger}$ ), 
but the solution procedure is well known [4]: One solves

$$
M v_{i}=m_{i} v_{i}^{*},
$$

where $m_{i}$ may always be chosen real and nonnegative, making the normalized vectors $v_{i}$ unique up to overall signs. In the nondegenerate case, one may obtain an equivalent solution by multiplying Eq. (3) on the left by $M^{*}$ :

$$
M^{*} M v_{i}=m_{i}^{2} v_{i}
$$

Here the $v_{i}$, which are now true normalized eigenvectors of $M^{*} M$, are defined only up to overall phases; nevertheless, since the neutrino mixing angles and Dirac phase $\delta$ are rephasing invariant, solving Eq. (44) produces all observables except the Majorana phases. Noting that $M^{*}=M^{\dagger}$ for complex symmetric matrices, one sees that $\left\{m_{i}^{2}\right\}$ are simply the modulus squares of the eigenvalues of $M$.

While the previous discussion is moot in the special case of exact TB mixing (since $U_{\mathrm{TB}}$ is real), it is relevant for studying models that deviate from the TB limit. Using the explicit TB form Eq. (11), it is easy to check that all diagonal matrices are in a one-to-one correspondence with mass matrices $M$ of the form

$$
M=\left(\begin{array}{ccc}
x & y & y \\
y & x+v & y-v \\
y & y-v & x+v
\end{array}\right)
$$

with complex eigenvalues $x-y, x+2 y$, and $x-y+2 v[6]$. This form has three independent complex degrees of freedom, $x, y$, and $v$, whereas the most general $3 \times 3$ complex symmetric matrix, has six parameters. The masses $m_{1,2,3}$ in normal form are the absolute values of these eigenvalues; $M$ in this form produces Majorana phases in $U_{\mathrm{MNS}}$, as discussed below.

The unique parametrization for the three remaining parameters, which we call $\epsilon, \delta_{1}$, and $\delta_{2}$, such that introducing each one of them in turn and keeping the other two zero precisely preserves one of the vectors forming the columns of $U_{\mathrm{TB}}$, is given by

$$
M=\left(\begin{array}{ccc}
x & y+\epsilon & y+2 \delta_{1} \\
y+\epsilon & x+v-\delta_{1} & y-v+\delta_{2} \\
y+2 \delta_{1} & y-v+\delta_{2} & x+v+\epsilon+3 \delta_{1}
\end{array}\right)
$$


The six parameters are given by

$$
\begin{aligned}
x & =M_{11}, \\
y & =+\frac{1}{3}\left(M_{12}+2 M_{13}+M_{22}-M_{33}\right), \\
v & =-\frac{1}{6}\left(6 M_{11}+M_{12}-M_{13}-5 M_{22}-M_{33}\right), \\
\epsilon & =+\frac{1}{3}\left(2 M_{12}-2 M_{13}-M_{22}+M_{33}\right), \\
\delta_{1} & =-\frac{1}{6}\left(M_{12}-M_{13}+M_{22}-M_{33}\right), \\
\delta_{2} & =-\frac{1}{2}\left(2 M_{11}+M_{12}+M_{13}-M_{22}-2 M_{23}-M_{33}\right) .
\end{aligned}
$$

This starting point, a fully general parametrization of $M_{L L}$ in terms of all possible deviations from TB mixing, is also the starting point in Ref. [5], which discusses the preservation of TB eigenvectors. Here, however, the preservation of TB eigenvectors is used as an organizing principle in defining the parametrization. It is straightforward to check that the unique choice of parameters $\delta_{1}, \epsilon$, and $\delta_{2}$ preserves the vectors given by the first, second, and third columns of $U_{\mathrm{TB}}$ in Eq. (1), respectively. In other words, $\epsilon \neq 0$ but $\delta_{1}=\delta_{2}=0$ in this parametrization preserves trimaximality, while $\delta_{1}, \delta_{2}$ parametrize all possible departures from it, and $\delta_{2} \neq 0$ but $\epsilon=\delta_{1}=0$ ( $\mu-\tau$ symmetry) preserves bimaximality, while $\epsilon$ and $\delta_{1}$ parametrize all possible departures from it. Introducing $\delta_{1} \neq 0$ but $\epsilon=\delta_{2}=0$ preserves the remaining orthogonal direction $(2,-1,-1)^{T}$.

Parametrizing $M$ as in Eq. (6) rather than $U_{\mathrm{TB}}$ is more convenient in the construction of flavor models, which typically provide textures of elements for Yukawa and Majorana matrices rather than the matrices that diagonalize them. Of course, $M$ here refers to the Majorana mass matrix $M_{L L}$ in the mass basis for the charged leptons (effectively, taking $Y_{L}$ diagonal). In models that specify nondiagonal forms for both $Y_{L}$ and $M_{L L}$, one faces the tricky question of why the mismatch between the matrices that diagonalize them nevertheless produces the TB angles to a high degree of accuracy.

The form of $M$ in Eq. (6) also allows for a straightforward calculation of the neutrino masses and mixing angles in a perturbative expansion simultaneously linear in $\epsilon, \delta_{1}$, and $\delta_{2}$, which is appropriate due to the closeness of the physical values to the TB solution. Solving 
Eq. (4) for the given $M$ produces the results

$$
\begin{aligned}
& m_{1}=\left|x-y-\frac{1}{2} \epsilon-\delta_{1}+\frac{1}{3} \delta_{2}\right|, \\
& m_{2}=\left|x+2 y+\epsilon+2 \delta_{1}+\frac{2}{3} \delta_{2}\right|, \\
& m_{3}=\left|x-y+2 v+\frac{1}{2} \epsilon+\delta_{1}-\delta_{2}\right|,
\end{aligned}
$$

and the corresponding modified eigenvectors

$$
\begin{gathered}
v_{1}=\frac{1}{\sqrt{6}}\left(\begin{array}{c}
2+2 \tilde{\delta}_{2}^{*} \\
-1-3 \tilde{\epsilon}^{*}+2 \tilde{\delta}_{2}^{*} \\
-1+3 \tilde{\epsilon}^{*}+2 \tilde{\delta}_{2}^{*}
\end{array}\right), \\
v_{2}=\frac{1}{\sqrt{3}}\left(\begin{array}{c}
1-2 \tilde{\delta}_{2} \\
1+3 \tilde{\delta}_{1}^{*}+\tilde{\delta}_{2} \\
1-3 \tilde{\delta}_{1}^{*}+\tilde{\delta}_{2}
\end{array}\right), \\
v_{3}=\frac{1}{\sqrt{2}}\left(\begin{array}{c}
-2 \tilde{\epsilon}+2 \tilde{\delta}_{1} \\
-1+\tilde{\epsilon}+2 \tilde{\delta}_{1} \\
1+\tilde{\epsilon}+2 \tilde{\delta}_{1}
\end{array}\right),
\end{gathered}
$$

where

$$
\begin{aligned}
\tilde{\epsilon} & \equiv \frac{1}{4} \frac{\operatorname{Re}\left[\epsilon(x-y+v)^{*}\right]-i \operatorname{Im}\left(\epsilon v^{*}\right)}{\operatorname{Re}\left[v(x-y+v)^{*}\right]} \\
\tilde{\delta}_{1} & \equiv-\frac{\operatorname{Re}\left[\delta_{1}(2 x+y+2 v)^{*}\right]+i \operatorname{Im}\left[\delta_{1}(3 y-2 v)^{*}\right]}{\operatorname{Re}\left[(3 y-2 v)(2 x+y+2 v)^{*}\right]} \\
\tilde{\delta}_{2} & \equiv \frac{1}{9} \frac{\operatorname{Re}\left[\delta_{2}(2 x+y)^{*}\right]-3 i \operatorname{Im}\left(\delta_{2} y^{*}\right)}{\operatorname{Re}\left[y(2 x+y)^{*}\right]}
\end{aligned}
$$

In models in which all the parameters are real, the combinations in Eq. (10) simplify to $\tilde{\epsilon}=\epsilon / 4 v, \tilde{\delta}_{1}=-\delta_{1} /(3 y-2 v)$, and $\tilde{\delta}=\delta_{2} / 9 y$. In this case, the independence of the eigenvectors on $x$ follows simply from the fact that it enters $M$ as a multiple of the identity matrix.

These expressions also elucidate the precise conditions for small departures from the TB form: $|\tilde{\epsilon}|,\left|\tilde{\delta}_{1}\right|,\left|\tilde{\delta}_{2}\right| \ll 1$. One can easily check that the pathological cases, e.g., $v=0$ but $\epsilon \neq 0$, lead to degenerate masses, and $U_{\text {MNS }}$ is no longer guaranteed to assume a TB form at leading order.

Now, taking into account the overall phase of $v_{i}$ appearing in the solution of Eq. (3) but not Eq. (4), one assembles the new MNS matrix

$$
U_{\mathrm{MNS}}=\left(v_{1}\left|v_{2}\right| v_{3}\right) P
$$


where $P \equiv \operatorname{diag}\left\{\exp \left(-\frac{i}{2} \operatorname{Arg} \mu_{i}\right)\right\}$, with $\mu_{i}$ being the complex mass parameters in Eq. (8)): $m_{i} \equiv\left|\mu_{i}\right| . \quad U_{\text {MNS }}$ reduces to TB form with nontrivial Majorana phases when $\tilde{\epsilon}, \tilde{\delta}_{1}$, and $\tilde{\delta}_{2}$ are set to zero; however, one also notes a different phase convention from Eq. (2), in which $U_{\mathrm{MNS}, 23}$ and $U_{\mathrm{MNS}, 33}$ are manifestly real. Multiplication of $U_{\mathrm{MNS}}$ on the left by an arbitrary diagonal phase matrix (i.e., rephasing its rows $U_{\mathrm{MNS}, i j} \rightarrow e^{i \theta_{i}} U_{\mathrm{MNS}, i j}$ ), which changes none of the observables, resolves this discrepancy. To first order in $\tilde{\epsilon}, \tilde{\delta}_{1}$, and $\tilde{\delta}_{2}$, one obtains the Euler angles

$$
\begin{aligned}
& s_{13}=\sqrt{2}\left|\tilde{\delta}_{1}-\tilde{\epsilon}\right|, \\
& s_{23}=\frac{1}{\sqrt{2}}\left|1-\tilde{\epsilon}-2 \tilde{\delta}_{1}\right| \simeq \frac{1}{\sqrt{2}}\left[1-\operatorname{Re}\left(\tilde{\epsilon}+2 \tilde{\delta}_{1}\right)\right], \\
& s_{12}=\frac{1}{\sqrt{3}}\left|1-2 \tilde{\delta}_{2}\right| \simeq \frac{1}{\sqrt{3}}\left(1-2 \operatorname{Re} \tilde{\delta}_{2}\right) .
\end{aligned}
$$

As expected, if bimaximality is preserved $\left(\epsilon=\delta_{1}=0\right)$, then $s_{13}=0$, and the atmospheric angle value is given by $s_{23}=1 / \sqrt{2}$ exactly. One also notes that $\delta_{2}$ alone in this approximation parametrizes departures from the solar angle value $s_{12}=1 / \sqrt{3}$. Using global fit values of the neutrino mixing angles [7],

$$
\begin{aligned}
& \sin ^{2} \theta_{13}=0.01_{-0.011}^{+0.016}, \\
& \sin ^{2} \theta_{23}=0.50_{-0.06}^{+0.07}, \\
& \sin ^{2} \theta_{12}=0.304_{-0.016}^{+0.022},
\end{aligned}
$$

one finds that the parameters are indeed small:

$$
\begin{aligned}
\left|\tilde{\delta}_{1}-\tilde{\epsilon}\right| & =0.07_{-0.04}^{+0.06}, \\
\operatorname{Re}\left(\tilde{\epsilon}+2 \tilde{\delta}_{1}\right) & =0.00_{-0.07}^{+0.06}, \\
\operatorname{Re} \tilde{\delta}_{2} & =0.023_{-0.017}^{+0.013} .
\end{aligned}
$$

The rephasing that leads to real $U_{\mathrm{MNS}, 23}$ and $U_{\mathrm{MNS}, 33}$ is

$$
\theta_{i}=\frac{1}{2} \operatorname{Arg}\left(\mu_{3}\right)+\eta_{i}, \text { where } \eta_{1,2,3}=0,+\operatorname{Im}\left(\tilde{\epsilon}+2 \tilde{\delta}_{1}\right),-\operatorname{Im}\left(\tilde{\epsilon}+2 \tilde{\delta}_{1}\right) .
$$

The MNS phases may then be obtained from Eqs. (91)-(11):

$$
\begin{aligned}
\delta & =-\operatorname{Arg}\left(\tilde{\delta}_{1}-\tilde{\epsilon}\right), \\
\beta_{1} & =\frac{1}{2}\left(\operatorname{Arg} \mu_{3}-\operatorname{Arg} \mu_{1}\right)-\operatorname{Im} \tilde{\delta}_{2}, \\
\beta_{2} & =\frac{1}{2}\left(\operatorname{Arg} \mu_{3}-\operatorname{Arg} \mu_{2}\right)-2 \operatorname{Im} \tilde{\delta}_{2},
\end{aligned}
$$


and the Jarlskog parameter is given by

$$
J=\frac{1}{3} \operatorname{Im}\left(\tilde{\delta}_{1}-\tilde{\epsilon}\right) .
$$

Note that $\beta_{1}, \beta_{2}$, and $\delta$ can all be nonzero even when $\tilde{\epsilon}, \tilde{\delta}_{1}, \tilde{\delta}_{2} \rightarrow 0$. Although the mass-squared splitting ratio $\Delta m_{21}^{2} / \Delta m_{32}^{2}=0.0319_{-0.0018}^{+0.0028}$ is experimentally small [7], it is nonvanishing in the TB limit:

$$
\frac{\Delta m_{21}^{2}}{\Delta m_{32}^{2}}=-\frac{3 \operatorname{Re}\left[y(2 x+y)^{*}\right]}{\operatorname{Re}\left[(3 y-2 v)(2 x+y+2 v)^{*}\right]}+O\left(\tilde{\epsilon}, \tilde{\delta}_{1}, \tilde{\delta}_{2}\right) .
$$

Discussion. A number of parametrizations of the MNS matrix have appeared in the literature [8]. Two recent parametrizations in particular describe deviations from TB in terms of three (real) parameters plus the CP-violating phase $\delta$. The relations of these two sets, $r, s$, and $a$ in Ref. [9] and $\epsilon_{13}, \epsilon_{21}$, and $\epsilon_{32}$ in Ref. [10], to those defined here at linear order are given by

$$
\begin{aligned}
\left|\tilde{\delta}_{1}-\tilde{\epsilon}\right| & =\frac{r}{2}=\frac{\epsilon_{13}}{\sqrt{2}}, \\
\operatorname{Re}\left(\tilde{\epsilon}+2 \tilde{\delta}_{1}\right) & =-a=-\epsilon_{32}, \\
\operatorname{Re} \tilde{\delta}_{2} & =-\frac{s}{2}=-\frac{\epsilon_{21}}{\sqrt{2}} .
\end{aligned}
$$

The advantages of the current parametrization have already been identified, but to summarize: (1) The parameters $\tilde{\epsilon}, \tilde{\delta}_{1}, \tilde{\delta}_{2}$ have a natural interpretation in terms of which TB eigenvector each preserves, even when the chosen parameter is not perturbatively small. (2) The mixing angles and Dirac phase of the MNS matrix have simple expressions at linear order in $\tilde{\epsilon}, \tilde{\delta}_{1}, \tilde{\delta}_{2}$. (3) Our parameters allow a direct analysis of mass matrix corrections that lead to a deviation from exact TB mixing in flavor models. For example, in one well-known model [11], the entries of $M_{L L}$ are determined by four vacuum expectation values (vevs) of Higgs triplets, labeled $a, b, c, d$. From the mass matrix given in Eq. (19) of Ref. [11], it is straightforward to check [using, for example, Eq. (7)] that

$$
\delta_{1}=\delta_{2}=0 \quad \text { and } \quad \epsilon=b-c
$$

Our parametrization shows immediately that this model is always exactly trimaximal. The deviation from bimaximality is reflected in $s_{13}=\frac{1}{2 \sqrt{2}}\left|\frac{b-c}{c-a}\right|$ when $|b-c| \ll|c-a|$ and all vevs are real. 


\section{Acknowledgments}

This work was supported by the NSF under Grant Nos. PHY-0757481 (CDC) and PHY0757394 (RFL).

[1] C. Amsler et al. [Particle Data Group], Phys. Lett. B 667, 1 (2008).

[2] P.F. Harrison, D.H. Perkins and W.G. Scott, Phys. Lett. B 530, 167 (2002) arXiv:hep-ph/0202074; B 535, 163 (2002) arXiv:hep-ph/0203209]; B 557, 76 (2003) arXiv:hep-ph/0302025]; Z.z. Xing, Phys. Lett. B 533, 85 (2002) arXiv:hep-ph/0204049];

L. Wolfenstein, Phys. Rev. D 18, 958 (1978); Y. Yamanaka, H. Sugawara and S. Pakvasa, Phys. Rev. D 25, 1895 (1982) [Erratum-ibid. D 29, 2135 (1984)].

[3] Z. Maki, M. Nakagawa, and S. Sakata, Prog. Theor. Phys. 28, 247; B.W. Lee, S. Pakvasa, R.E. Shrock and H. Sugawara, Phys. Rev. Lett. 38, 937 (1977) [Erratum-ibid. 38, 1230 (1977)].

[4] Variants on this result apprears to have been independently discovered multiple times. See, for example, L. Autonne, Annales de l'Université de Lyon, Nouvelle Série I, Fasc. 38 (1915) 1; T. Takagi, Japan J. Math. 1 (1925) 83; I. Schur, Am. J. Math. Phys. 67, 472 (1945); B. Zumino, J. Math. Phys. 3, 1055 (1962). A nice discussion of these issues appears in Appendix A of S.Y. Choi, H.E. Haber, J. Kalinowski, and P.M. Zerwas, Nucl. Phys. B 778, 85 (2007) arXiv:hep-ph/0612218.

[5] C.H. Albright and W. Rodejohann, Eur. Phys. J. C 62, 599 (2009) [arXiv:0812.0436 [hep-ph]]; Phys. Lett. B 665, 378 (2008) arXiv:0804.4581 [hep-ph]].

[6] G. Altarelli and D. Meloni, J. Phys. G 36, 085005 (2009) arXiv:0905.0620 [hep-ph]].

[7] T. Schwetz, M.A. Tortola, and J.W.F. Valle, New J. Phys. 10, 113011 (2008) arXiv:0808.2016 [hep-ph]].

[8] N. Li and B.Q. Ma, Phys. Rev. D 71, 017302 (2005) arXiv:hep-ph/0412126]; S.W. Li and B.Q. Ma, Phys. Rev. D 77, 093005 (2008) arXiv:0806.0670 [hep-ph]]; X.G. He, S.W. Li and B.Q. Ma, Phys. Rev. D 78, 111301 (2008) arXiv:0809.1223 [hep-ph]]; Phys. Rev. D 79, 073001 (2009) arXiv:0903.2880 [hep-ph]]; A. Datta, L. Everett and P. Ramond, Phys. Lett. B 620, 42 (2005) arXiv:hep-ph/0503222]; L.L. Everett, Phys. Rev. D 73, 013011 (2006) arXiv:hep-ph/0510256]; P. Kaus and S. Meshkov, AIP Conf. Proc. 672, 117 (2003) 
arXiv:hep-ph/0211338; A. Zee, Phys. Rev. D 68, 093002 (2003); arXiv:hep-ph/0307323; Z.z. Xing, J. Phys. G 29, 2227 (2003) arXiv:hep-ph/0211465; J.D. Bjorken, P.F. Harrison, and W.G. Scott, Phys. Rev. D 74, 073012 (2006) arXiv:hep-ph/0511201; W. Rodejohann, Phys. Lett. B 671, 267 (2009) arXiv:0810.5239 [hep-ph]]; W. Grimus and L. Lavoura, JHEP 0809, 106 (2008) arXiv:0809.0226 [hep-ph]]; Phys. Lett. B 671, 456 (2009) arXiv:0810.4516 [hep-ph]].

[9] S.F. King, Phys. Lett. B 659, 244 (2008) [arXiv:0710.0530 [hep-ph]].

[10] S. Pakvasa, W. Rodejohann, and T.J. Weiler, Phys. Rev. Lett. 100, 111801 (2008) arXiv:0711.0052 [hep-ph]].

[11] E. Ma, Phys. Rev. D 70, 031901 (2004) arXiv:hep-ph/0404199. 